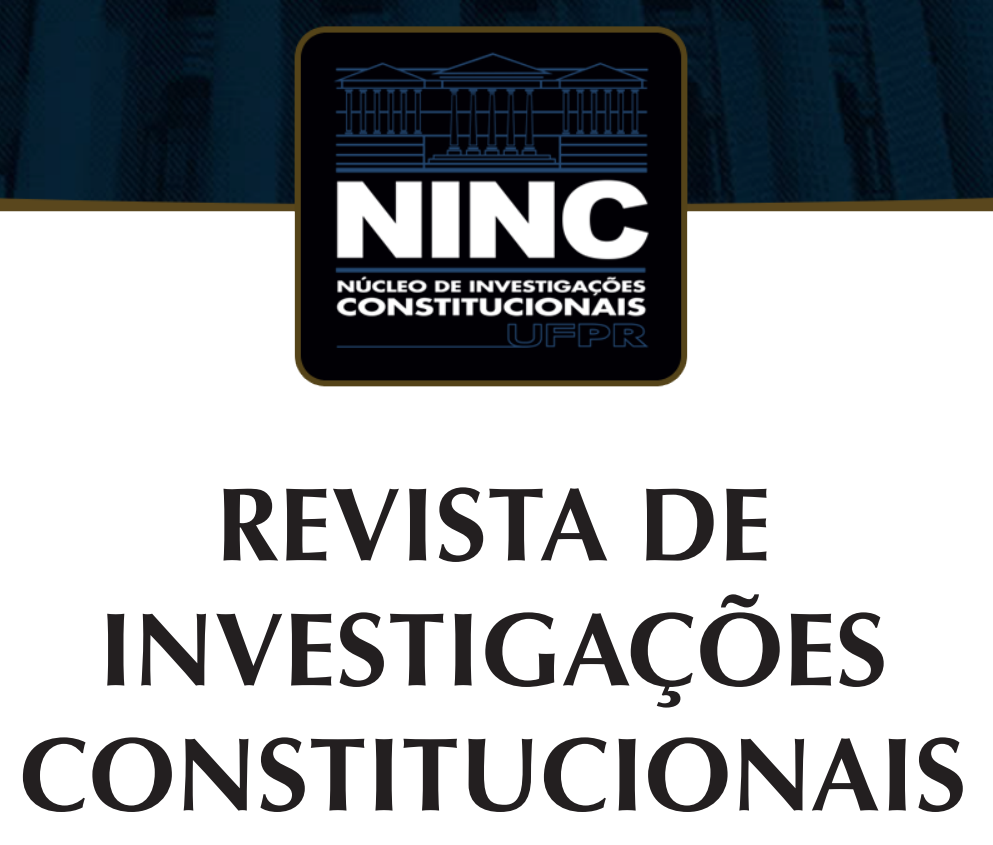

JOURNAL OF CONSTITUTIONAL RESEARCH

vol. 6 | n. 3 | setembro/dezembro 2019 | ISSN 2359-5639 | Periodicidade quadrimestral Curitiba | Núcleo de Investigações Constitucionais da UFPR | www.ninc.com.br 


\title{
Aspects de droit financier sous-jacents au processus d'impeachment au Brésil
}

\section{Financial law aspects underlying the impeachment process in Brazil}

\author{
HUGO DE BRITO MACHADO SEGUNDO I, II, * \\ ' Universidade Federal do Ceará (Fortaleza-CE, Brasil) \\ "Centro Universitário Christus - Unichristus (Brasil) \\ hugo.segundo@ufc.br \\ http://orcid.org/0000-0002-0687-9592
}

\section{RAQUEL CAVALCANTI RAMOS MACHADO ${ }^{1, * *}$}

' Universidade Federal do Ceará (Fortaleza-CE, Brasil) raquelramosmachado@gmail.com https://orcid.org/0000-0002-9749-3539

Recebido/Received: $18.04 .2018 /$ April $18^{\text {th }}, 2018$ Aprovado/Approved: 30.10 .2019 / October $30^{\text {th }}, 2019$

\section{Resumé}

Le processus d'impeachment de Dilma Rousseff, au Brésil, découle de l'adoption de conduites questionnables à la lumière du Droit Financier, de I'utilisation de banques publiques pour financer indirectement les dépenses publiques ("pédalage fiscal", acrobaties comptables), qu ont mené à un déséquilibre des comptes publics, avec des conséquences assez nuisibles pour l'économie nationale, lesquelles sont ressenties, dans le secteur public

\section{Abstract}

The impeachment process of Dilma Roussef, in Brazil, arises from the adoption of questionable decisions in the light of Financial Law, as the use of public banks to indirectly finance public expenditure ("tax pedaling", accounting acrobatics), which led to an imbalance in the public accounts, with consequences quite harmful for the Brazilian economy, which are felt, in the public sector and the private initiative, to the present day, and may continue for many years. In spite

Como citar esse artigo/How to cite this article: MACHADO SEGUNDO, Hugo de Brito; MACHADO, Raquel Cavalcanti Ramos. Aspects de droit financier sous-jacents au processus d'impeachment au Brésil. Revista de Investigações Constitucionais, Curitiba, vol. 6, n. 3, p. 647-667, set./dez. 2019. DOI: 10.5380/rinc.v6i3.57889.

* Professor Adjunto da Faculdade de Direito da Universidade Federal do Ceará e do Curso de Mestrado em Direito do Centro Universitário Christus - Unichristus (Fortaleza-CE, Brasil). Doutor em Direito pela Universidade de Fortaleza e Mestre em Direito pela Universidade Federal do Ceará. Coordenador do Grupo de Pesquisas sobre "Democracia e Finanças Públicas", junto à Faculdade de Direito da Universidade Federal do Ceará. Foi coordenador do Programa de Pós-Graduação (Mestrado/Doutorado) da Faculdade de Direito da Universidade Federal do Ceará (2012-2016). Membro do Instituto Brasileiro de Direito Tributário - IBDT e do Instituto Cearense de Estudos Tributários - ICET. Visiting Research Scholar na Wirtschaftsuniversität, Viena, Áustria (20122013 e 2015-2016).E-mail: hugo.segundo@ufc.br.

** Professora Adjunta e Chefe do Departamento de Direito Público da Universidade Federal do Ceará (Fortaleza-CE, Brasil). Doutora em Direito pela Universidade de São Paulo (São Paulo-SP, Brasil). Mestra em Direito pela Universidade Federal do Ceará (Fortaleza-CE, Brasil). Visiting Research Scholar da WirtschaftsUniversistat Vienna (2015 e 2016). Professora pesquisadora convidada da Faculdade de Direito da Universidade Paris Descartes (2017). E-mail: raquelramosmachado@gmail.com. 
et l'initiative privée, jusqu'à nos jours, pouvant continuer durant de nombreuses années. On ne peut nier, malgré tout, l'aspect polémique et limitrophe de l'encadrement de ces ilégalités comme crime de responsabilité fiscale, raison pour laquelle on ne peut qualifier d'absurdes ou d'inopportunes les points de vue de ceux qui reconnaissent la présence de conditions techniques pour l'impeachment, comme de ceux qui les rejettent. De quelque manière que ce soit, le fait que la décision ait été prise par l'organisme compétent pour cela, sous les lumières du texte constitutionnel, avec le contrôle, en ce qui concerne les aspects formels, du Suprême Tribunal Fédéral, et à partir des constatations du Tribunal des Comptes et du Ministère Public des Comptes, nous montre qu'il n'est pas approprié d'affirmer qu'il y a eu un "coup d'État", vu que tout s'est passé dans les termes prévus par le texte constitutionnel.

Mots-clés: impeachment; Droit Financier; brésilien; pédalage fiscal; crédits supplémentaires. of everything, we cannot deny the controversial and bordering aspect of the framing of these illegalities as a crime of fiscal responsibility, which is why we cannot consider as "absurd" the views of those who recognize the presence of technical conditions for impeachment, as well as those who reject them. In any way, the fact that the decision was made by the competent organ - Congress -, under the light of the Constitution, with the control, regarding to the formal aspects, of the Supreme Federal Court, and from findings of the Court of Auditors and the Public Ministry of Accounts, shows us that it is not appropriate to affirm that there was a "coup d'etat", since everything happened in the terms provided by the Constitution.

Keywords: impeachment; Financial Law; Brazilian; tax pedaling; additional credits.

\section{SOMMAIRE}

1. Introduction; 2. Le processus d’impeachment et les Conduites imputées à la Présidente; 2.1 Contracter des emprunts auprès de banques publiques; 2.2 Décrets d'ouverture de crédits supplémentaires sans autorisation législative; 2.3 Autres aspects de la demande non- admise par le Président de la Chambre des Députés; 3. Arguments utilisés pour sa défense; 3.1 Quant à l'opération de crédit avec les banques privées; 3.2 Quant à la création de crédits supplémentaires; 4. Adoption de la pratique par d'autres Présidents, l'accord du TCU et la configuration comme " opération de crédit"; 5 . Les décrets d'ouverture de crédits supplémentaires; 6 . Principe de la compétence, caractère public de la décision et possibilité de critique de la décision prise par un organisme compétent; 7 . Conclusions; 8 . Références.

\section{INTRODUCTION}

Initié en 2015 et conclu en août 2016, le processus de mise en accusation (impeachment) de la Présidente Dilma Rousseff, au Brésil, a suscité, et suscite encore, maintes questions, tant sur le plan politique que sur le plan judiciaire. Dans ce contexte, le manque d'une connaissance plus approfondie sur les infractions lui étant imputées, à la lumière du Droit Financier, et les aspects politiques et idéologiques sous-jacents à la question, ont servi d’ingrédients pour diviser la société brésilienne en un débat dans lequel il y a souvent peu de rationalité, où prédomine la répétition émotive de mots d'ordre des deux côtés.

Dans ce travail, nous prétendons examiner le fondement juridique de la demande de mise en accusation, à la lumière des normes brésiliennes du Droit Financier. Nous espérons, avec cela, jeter des lumières qui aident à comprendre le processus. L'analyse, relève-t-on, se fera uniquement sur les aspects juridiques, précisément parce que ce 
sont ceux autour desquels il y a la moindre connaissance du public en général, et au sujet desquels les auteurs de ce travail peuvent, peut-être, apporter quelque contribution. Pour cette raison, les questions politiques ne seront pas abordées- même si elles sont importantes dans un procès de cette nature- liées, par exemple, à de probables manouvres du Vice-Président en faveur de l'impeachment, ou à la convenance des mesures adoptées par le Gouvernement de Dilma dans le domaine économique, ou encore à la participation de son parti et de membres de son gouvernement dans des scandales de corruption. Ces questions d'ordre politique ne seront citées que pour mieux contextualiser le scénario confus dans lequel le processus s'est déflagré.

L'objectif du texte, en somme, est d'examiner les fondements juridiques invoqués pour justifier la mise en accusation, de manière à juger si la décision du Sénat Fédéral est restée confinée à l'intérieur de sa compétence constitutionnelle, ou non. Dans le cas affirmatif, relève-t-on, critiquer la décision continuera d'être légitime, puisqu'on est en désaccord avec elle, soit pour des questions politiques, soit même pour des divergences dans l'interprétation des normes juridiques applicables. En fin de compte, il est toujours possible de critiquer des décisions, même quand elles sont prises par un organisme d'état dans l'exercice d'une compétence attribuée par la Constitution. Mais le moindre désaccord entre le mérite de la décision prise par l'organisme compétent et la compréhension de qui éventuellement la critique ne constitue pas un motif, en soi, pour se dire qu'il y a eu un» coup d'état» ou que les institutions se sont rompues.

Le travail sera divisé en trois sujets au terme desquels nous présenterons la conclusion. Dans le premier sujet, nous présenterons les arguments de l'accusation, un moment où nous mènerons l'étude sur le concept d'impeachment et sur les faits pouvant conduire à son apparition. La question sera contextualisée en prenant compte des normes juridiques brésiliennes constitutionnelles et infra-constitutionnelles concernant le Droit Financier. Dans le deuxième sujet, nous ferons des pondérations envers les arguments de défense, et sur le défi interprétatif quant à la signification des normes invoquées par l'accusation. Pour ce qui est du troisième sujet, nous établierons une analyse comparative avec les conduites semblables d'autres présidents, questionnant en outre la compétence du Sénat pour prendre des décisions sur toutes les questions qui lui ont été posées.

\section{LE PROCESSUS D'IMPEACHMENT ET LES CONDUITES IMPU- TÉES À LA PRÉSIDENTE}

L'impeachment est une forme de contrôle du Pouvoir Législatif par rapport à certains pouvoirs, entre lesquels, principalement, celui du chef de l'Exécutif ${ }^{1}$, et il dé-

BONAVIDES, Paulo. Ciência Política. 10 ed. São Paulo: Malheiros, p. 141. 
tient un caractère juridique et politique ${ }^{2}$. L'aspect juridique est mis en évidence devant la prévision de conduites spécifiques pouvant conduire à la sortie du Chef de l'Exécutif du pouvoir, dans le modèle présidentialiste. Autrement dit, ce n'est pas la simple décision politique d'écarter le gouvernant qui est la raison d'être de l'impeachment, mais plutôt la pratique de conduites imputées en tant que «crime de responsabilité» par l'ordonnance juridique ${ }^{3}$. En revanche, son caractère politique découle du fait qu'il peut être déflagré par n'importe quel citoyen, en vertu de son statut de participant de l'État, et non obligatoirement initié par un organisme d'État, comme c'est le cas pour les crimes prévus dans les lois pénales. En outre, après la déflagration du processus, un concours d'intérêts entre en scène, et la gouvernabilité du pays, comme aussi la force politique du gouvernant, finissent par influencer le procès. Le respect des aspects juridiques de l'impeachment lui donne plus de justice et de légitimité, mais son caractère politique lui fournit plus de pouvoirs de décision, en attribuant par ailleurs aux juges une liberté de justification, lesquels auront seulement à répondre par oui ou par non quant à l'existence d'un crime de responsabilité, en délibérant sur l'application de la sanction.

Le jugement sera réalisé par le Sénat Fédéral, dans les termes de l'article 52 de la Constitution de la République Fédérative du Brésil (CF/88), mais sera présidé par le président du Suprême Tribunal Fédéral, exactement comme un moyen d'assurer une ample défense et son contradicteur. Avant cela, cependant, la Chambre des Députés devra recevoir l'accusation, par deux tiers de ses membres.

D’après l'art. 85 de la Constitution Fédérative du Brésil(CF/88), les actes du Président de la République allant à l'encontre de la Constitution Fédérale sont des crimes de responsabilité et, spécialement, contre: (i) le libre exercice du Pouvoir Législatif, du Pouvoir Judiciaire, du Ministère Public et des Pouvoirs constitutionnels des unités de la Fédération; (iii) l'exercice des droits politiques, individuels et sociaux; (iv) la sécurité interne du Pays; (v) la probité dans I'administration; (vi) la loi de finances; (vii) I'application de la loi et des décisions judiciaires. En accord, encore, avec la Constitution, une loi spécifique doit définir de tels crimes et réglementer le processus et le jugement inhérents à son imputation au Président.

Nous percevons qu'un « crime de responsabilité» n'est pas comparable à un crime commun, celui-ci étant défini dans le domaine du Droit Pénal. Les «crimes de responsabilité sont définis constitutionnellement comme étant ceux qui intentent contre

2 Cf. SILVA, Carol; JENKINS-SMITH, Hank; WATERMAN, Richard. Why Did Clinton Survive the Impeachment Crisis? A Test of Three Explanations. Presidential Studies Quarterly, Washington, v. 37, n. 3, p. 468-485, Sep. 2007, p. 468. L'objective de l'impeachment est d'éviter à la fois une dictature de l'Exécutif et du Législatif. Par conséquent, il n'est pas admis en relation avec des actes de la vie privée du Président de la République, mais avec des actes liés à la manière dont il exerce ses fonctions. Cf. v.g., ARATO, Andrew. Impeachment or revision of the Constitution? Constellations, Massachusetts, v. 6, n.2, 145-156, 1999, p. 146 e ss.

3 BONAVIDES, Paulo. Ciência Política. 10 ed. São Paulo: Malheiros, p. 313. 
I'un des biens décrits dans l'art. 85 de la CF/88, étant définis plus en détail par la loi 1.079, de 1950, avec un diplôme reçu par la Constitution le désignant comme celui qui s'occupe de tels crimes, comme également du procès et du jugement qui lui sont liés.

Pour ce qui est de Dilma, on lui a imputé deux conduites qui pourraient configurer un crime de responsabilité, savoir: (i) contracter des emprunts auprès de banques publiques; et (ii) l'édition de décrets permettant des crédits supplémentaires, sans autorisation législative. Pour les auteurs de la pièce d'accusation, de telles conduites seraient contraires à la loi de finances, l'un des biens juridiques référés dans l'article 85 de la CF/88, et seraient caractérisées comme crimes de responsabilité dans la Loi nº $1.079 / 50$.

\subsection{Contracter des emprunts auprès de banques publiques}

Selon l'article 136 de la loi complémentaire n 101/2000, il est «interdite l'opération de crédit entre une institution financière d'État et la personne de la Fédération qui la contrôle, dans la qualité de bénéficiaire de l'emprunt». On prétend, avec cela, interdire à la personne publique, parce qu'elle est aussi celle qui contrôle l'institution financière, qu'elle en obtienne des emprunts de forme illimitée, sans contrôle ou modération, chose qui n'arriverait pas si l'emprunt était sollicité auprès de l'institution financière privée indépendante, non contrôlée par le propre preneur de l'emprunt.

En dehors du fait d'être un important mécanisme pour contenir l'endettement public, qui évite toutes les conséquences négatives qu'il est capable d’apporter aux finances publiques et à l'économie en général, l'interdiction a une explication historique. Dans les années 1980 et 1990, de nombreuses banques d'états ont été supprimées par les gouvernements qui les contrôlaient, lesquels en obtenaient des financements quasiment illimités. Outre le fait que la dette des états-membres ait pris des proportions astronomiques, quelque chose que la Loi Complémentaire 101/2000 voulait éviter, cette pratique a décimé ces banques. Cela sans parler du risque encouru par les propres associés qui, pendant qu'ils croient que leur argent est correctement investi par la banque, celui-ci, en réalité, est utilisé pour financer les dettes de l'État. D’où l'interdiction.

Dans le cas de la Présidente Dilma Rousseff, il n'y a pas eu, de forme explicite, solennelle et formelle, la célébration d'un contrat d'emprunt, car justement la loi le défend expressément. Toutefois le Gouvernement Fédéral_attribue aux banques publiques - Caixa Econômica Federal, Banco do Brasil, et Banco Nacional de Desenvolvimento - BNDES - la fonction de payer des obligations qui lui reviennent en tant que gouvernement. Des bénéfices d'assistance de programmes comme «bourse famille», «ma maison, ma vie», «assurance-chômage» et «crédit agricole», par exemple. D’après ce système, les banques devraient seulement opérationnaliser la remise des valeurs à 
leurs bénéficiaires, avec la certitude que, avant que les paiements soient effectués, le gouvernement transférait aux banques les valeurs nécessaires.

Le fait est que, à partir de 2014, le gouvernement a commencé à retarder, de manière systématique, le paiement de ces valeurs aux banques qu'il contrôlait, lesquelles, pourtant, payaient ponctuellement les bénéficiaires respectifs. Grâce à cela, la banque honorait une dette du gouvernement, et il lui restait un crédit auprès de celui-ci. Voilà pourquoi on a affirmé que cette pratique correspondait, même étant maquillée ou déguisée, à une opération de crédit, interdite par l'art. 36 de la LC 101/2000. Ce fut pour cette pratique que l'on attribua le surnom de «pédalage fiscal» (acrobaties comptables), car, grâce à lui, la Présidente aurait donné une plus grande «impulsion» aux comptes publics, en les montrant plus sains qu'ils n'étaient en réalité.

\subsection{Décrets d'ouverture de crédits supplémentaires sans autorisa- tion législative}

La deuxième infraction imputée à la Présidente Dilma a été l'édition de décrets par lesquels on a autorisé de nouveaux crédits supplémentaires dans le budget, sans qu'il y ait autorisation législative pour cela.

Il est besoin de rappeler que le budget contient la prévision des recettes et des dépenses, et il n'est pas rare que les prévisions ne se réalisent pas. Les recettes peuvent ne pas correspondre à l'estimation initiale, par exemple, en vertu d'une crise économique, ou d'une décision judiciaire qui affirme un tribut indû dont la collecte était attendue. Les dépenses, pourtant, doivent respecter la limite prévue, raison pour laquelle, afin de prévenir de tels imprévus, le Législatif autorise au préalable le Président de la République, en cas de nécessité, à prendre des crédits supplémentaires, jusqu'à une limite préfixée, et si les conditions légalement établies sont remplies.

Pour ce qui est de Dilma Rousseff, la loi de finances (celle de 2014 comme celle de 2015) conditionne l'ouverture de crédits supplémentaires lorsque l'objectif de l'excédent primaire est atteint par le Gouvernement Fédéral. Dit autrement, il faudrait au Gouvernement des recettes qui dépassent ses dépenses, d'où seront exclus seulement les intérêts de la dette (ce qui est connu comme l'excédent primaire), pour que la Présidente puisse, par décret, prendre des crédits supplémentaires.

Ce qui s'est passé, toutefois, fut que la Présidente édita des crédits supplémentaires, même n'ayant pas atteint l'objectif de l'excédent primaire exigé comme condition par la Loi de Finances. Ainsi, I'infraction serait configurée. 


\subsection{Autres aspects de la demande non-admise par le Président de la Chambre des Députés}

II vaut la peine de noter que la demande de mise en accusation garde encore un contenu plus ample, en imputant à la Présidente des infractions liées à sa possible participation dans les scandales de corruption de l'entreprise d'état d'exploitation du pétrole, la Petrobrás, dont le Conseil d'Administration a été présidé par Dilma et où elle a participé à l'époque dans laquelle des opérations litigieuses se sont déroulées, à l'exemple de celles liées à la négociation d'une Raffinerie à Pasadena, aux États-Unis. On lui imputait, en plus, des infractions relatives à son premier mandat comme Présidente de la République. Chacune d'entre elles, cependant, a été rejetée par le Président de la Chambre des Députés ${ }^{4}$, Eduardo Cunha, car il ne s'agissait pas de faits pratiqués dans l'exercice du mandat présidentiel dont on cogite la mise en accusation.

De toute façon, il semble que l'on ne puisse nier qu'ils ont influencé, dans le domaine politique, le jugement correspondant, comme on le perçoit dans le propre discours de beaucoup de parlementaires qui ont participé au processus.

\section{ARGUMENTS UTILISÉS POUR SA DÉFENSE ${ }^{5}$}

Durant sa défense, Dilma Rousseff n'a rejeté aucun des faits qui lui avaient été imputés dans la demande d'impeachment, dans la forme reçue par la Chambre des Députés. Elle n'a pas nié le fait que les banques publiques aient payé des dettes du Gouvernement Fédéral, lequel les a soldées après un certain temps. Elle n’a pas nié non plus l'édition de décrets d'ouverture de crédits supplémentaires. Sa réfutation s'est faite, spécifiquement, sur la signification juridique de tels faits.

\subsection{Quant à l'opération de crédit avec les banques privées}

La défense de Dilma Rousseff a affirmé, quant au «pédalage fiscal» (acrobaties comptables), qu'il n'y aurait pas eu d'opération de crédit avec des banques publiques, car aucun contrat n'a été signé, formel et écrit, avec de telles banques, pour motiver

\footnotetext{
4 Au Brésil, où le parlement adopte le système des deux chambres, la demande de mise en accusation a son admission appréciée par la Chambre des Députés, et, ensuite, reçue par elle, est jugée par le Sénat Fédéral, qui fait un nouveau jugement d'admission, et, ensuite, si c'est le cas, passe à l'analyse des mérites. Ce rituel a été défini par le Suprême Tribunal Fédéral, dans le jugement de l'argumentation de Désobéissance au Précepte Fondamental (ADPF) 378, réalisée par le Parti Communiste du Brésil - PCdoB, à partir d'une interprétation de la Loi 1.079/50 à la lumière de la Constitution Fédérale de 1988.

5 Le document principal qui a été le fondement de la défense de Dilma a été un avis élaboré par le Professeur Ricardo Lodi Ribeiro, de I'Université de l'État de Rio de Janeiro, dont les fondements sont synthétisés dans cet article. Pour la teneur complète de l'avis, voir <http://s.conjur.com.br/dl/parecer-ricardo-lodi-impeachment. pdf $>$. En dehors du document en question, qui du point de vue technique est que le meilleur organise les arguments en défense du Président, tous les discours et manifestations en défense de Dilma Roussef, d'elle-même et de son avocat, José Eduardo Cardozo, sont disponibles dans <www.youtube.com >.
} 
un crédit de celles-ci envers I'Union. De forme différente, la dette de I'Union envers les banques découlerait de la loi, qui a attribué aux banques la fonction de réaliser les paiements et à l'Union l'obligation de leur transférer les ressources correspondantes. Ainsi, le « crédit» que la banque aurait vis à vis de l'Union serait équivalent, par exemple, au crédit qu'un contribuable, qui paie un tribut indû, aurait vis à vis d'elle, pour ce qui se rapporte à la respective restitution. Comme il s'agit de l'imputation d'un «crime», l'interprétation de la loi devrait être stricte, l'interprète ne devant pas augmenter la signification de l'expression « opération de crédit», qui aurait une signification claire et définie dans l'ordre juridique.

D’un autre côté, même pouvant être considérée illégale, la pratique de «pédalage» (acrobaties comptables) serait contraire à la Loi de Responsabilité Fiscale, et non pas à la Loi de Finances. En accord avec l'art. 85 de la Constitution, le crime se caractérise par la pratique d'un acte outrageant envers la Loi de Finances, une entité qu'on ne pourrait confondre avec la Loi Complémentaire 101/2000, qui est une norme du Droit Financier destinée à imposer aux gestionnaires de la responsabilité dans l'administration des deniers publics. Dans la loi de finances, en soi, il n'y aurait aucune disposition contrariée par la pratique en question.

Même si la Loi 1.079/50 définit comme crime de responsabilité, aussi, des actes liés à la «garde et à l'emploi légal de l'argent public», énumérant, de forme spécifique, l'action de « contracter un emprunt, émettre de la monnaie courante ou des actions, ou effectuer une opération de crédit sans autorisation légale» (art. 11, item 3), cette partie de la loi n'aurait pas été reçue par la Constitution de 1988, vu que l'art. 85 de la Constitution contient une relation moins longue dans l'énumération des biens juridiques sauvegardés dans la définition des crimes de responsabilité, dans laquelle on ne prévoit pas la «garde et l'emploi légal de l'argent public» prévue par la loi 1.079/50.

En outre, la pratique du «pédalage» (acrobaties comptables) n'aura pas été inaugurée par Dilma Rousseff. Maints Présidents antérieurs avaient déjà fait la même chose, et le Tribunal des Comptes de I'Union(TCU) n'avait jamais désapprouvé leurs comptes à cause de cela. Avec Dilma, en dehors du fait d'avoir altéré de forme surprenante sa jurisprudence, le Tribunal l'a fait de manière rétroactive, la punissant pour des comportements qui jusqu'à lors n'étaient pas considérés des infractions. Et cela sans se référer au fait que ces ressources auraient été utilisées pour honorer des dépenses d'assistance aux plus démunis, liés au droit fondamental d'une parcelle significative de la population brésilienne à la subsistance («bourse famille» et «assurance chômage») et au logement («ma maison ma vie»). 


\subsection{Quant à la création de crédits supplémentaires}

Quant à la création de crédits supplémentaires, la Présidente reconnait de fait avoir édité de tels décrets, même si elle n'a pas répondu à la condition légalement exigée pour cela, qui était le respect de l'objectif de l'excédent primaire prévu par la Loi des Directives Budgétaires (LDO) pour les années 2014 et 2015. Elle a donc argumenté sa défense, cela a eu lieu à cause de la grave crise qui a dévasté l'économie brésilienne, qui a compromis ses recettes tributaires fédérales. Et encore: aussitôt qu'il fut évident que l'objectif ne serait pas atteint, le Pouvoir Exécutif Fédéral sollicita, auprès du Congrès National, la révision de l'objectif prévu à la LDO, ce qui fut fait. Dans cet ordre d'idées, devant le nouvel objectif fiscal, revu par le Congrès en 2015, les décrets d'ouverture de crédits seraient «régularisés», puisque le gouvernement réaliserait le nouvel objectif et, ainsi, serait autorisé à les éditer.

\section{ADOPTION DE LA PRATIQUE PAR D AUTRES PRÉSIDENTS, L'AC- CORD DU TCU ET LA CONFIGURATION COMME "OPÉRATION DE CRÉDIT»}

Quant à l'argument selon lequel d'autres présidents auraient été inclus dans la même pratique de retarder les versements aux banques publiques qui réalisent des paiements de dettes de l'Union, ceux favorables à l'impeachment ont argumenté qu'une «erreur n'en justifiait pas une autre», parce que, si la pratique est une faute, le fait d'avoir été adoptée par des présidents antérieurs ne la rendrait pas légitime quand elle le sera par Dilma Rousseff. Au contraire, si le TCU, par manque de structure et d'outillage, ne s'apercevait pas immédiatement d'une irrégularité, restant en silence devant elle dans un premier temps, jamais plus elle ne pourrait prendre position contre elle, un argument qui d'ailleurs fit partie du vote du Ministre João Augusto Ribeiro Nardes (TC 005.335/2015).

Cependant, même que la pratique eut été expressément plébiscitée par le Tribunal des Comptes en des situations antérieures, ce qui ne fut pas le cas, il est important de comparer ce que les présidents antérieurs ont fait, et ce qui est imputé à Dilma Rousseff. L'importance, la durée et la systématicité des «pédalages» (acrobaties comptables) peut, en principe, justifier un changement de compréhension par rapport à eux.

Vraiment, si un Président retarde, pour quelques jours, le paiement de quelques millions, on peut comprendre qu'il y a eu un simple défaut d'obligation légale de l'Union auprès des banques, et non pas une «opération de crédit». Cependant, si le retard dure plusieurs mois, et qu'il a une intensité de plusieurs milliards, on peut en conclure que, par des voies biaisées, le gouvernement cherche des financements auprès des banques publiques, avec tous les maléfices qui en découlent, et ce qui contrarie l'art. 36 de la LC 
$101 / 2000$. Cette conclusion se renforce quand on en vient à l'observation que les ressources sont définitivement remises aux banques quand ainsi le détermine le TCU, ce qui conduit à la constatation que, s'il n'y avait pas d'objection de la Cour des Comptes, le financement aurait continué de manière incontrôlée, justement comme la LRF vise à l'éviter. Et ce n'est non seulement pas ce qui est arrivé, mais encore, après que le TCU ait condamné la pratique, les comptes publics ont dû être refaits, ce qui généra un déficit monumental.

Le remake des comptes publics, à ce propos, a été dû au fait que le «pédalage» (acrobaties comptables) permettait, par ailleurs, une falsification des données référents, car la dette de I'Union auprès des banques publiques n'apparaissait pas sur les comptes en question, lesquelles, cependant, soldaient des dettes fédérales. Cela signifie que, en dehors de s'être fait financer auprès des banques publiques, le gouvernement Dilma Rousseff I'a fait de forme maquillée, sans que cela soit mis dans les comptes publics de forme transparente, qui ont montré un résultat entièrement irréel. Cette impression qui montrait les comptes relativement équilibrés a été divulguée lors de sa réélection, ce qui a certainement contribué à la rendre plus légitime.

Nous pouvons dire que le graphique ci-dessus ne reflète guère l'inflation de cette période, ni la différence de l'importance du budget de Dilma et de ses prédécesseurs. Mais même avec ces ajustements, la différence continue significative, qui a également continué en 2015, ayant été interrompue seulement au moment de l'objection faite par le Tribunal des Comptes. Ce fut la continuité, dans I'année 2015, qui a rendu possible le jugement de Dilma à cause de cette conduite, même si elle ait été plus accentuée en 2014.

C'est toujours l'importance, la durée et la systématisation du « pédalage» (acrobaties comptables) qui permet, peut-être, de dire qu'il configure une forme "simulée», ou «en fraude face à la loi», de réaliser l'opération de crédit que la loi prohibe. Au lieu de signer un contrat, Dilma a obtenu, de façon biaisée, toujours le même résultat, ayant la même intention, qui seraient inhérents à ce contrat. Un déficit public significatif a été occulté, lequel a été financé par les banques publiques de forme interdite par la loi, mais par des chemins ou par des voies ayant une apparence de validité.

D'ailleurs, les figures représentantes de l'abus de droit, de la simulation et de la fraude à la loi, communément invoquées par la Finance Publique pour déconsidérer des affaires pratiquées par des contribuables dans la sphère de la « planification fiscale», sont parfaitement applicables ici. Par une voie anormale et apparemment légitime, on a fait exactement ce que la loi interdit, avec l'intention (financement de l'État) que la loi interdit, en atteignant le résultat que la loi interdit.

Il y a simulation quand le sujet désire pratiquer l'acte «a», lorsqu'il pratique effectivement l'acte «a», mais qu'il déclare formellement la production du fait «b». C'est le cas d'un débiteur qui transfère formellement la titularité de ses biens à un tiers, 
une personne interposée, mais qui continue, de fait, d'être son possesseur, en exerçant toutes les facultés d'un propriétaire, et le faisant uniquement pour échapper à un procès d'exécution. Un exemple est celui de l'employé qui propose, à la demande du patron, une réclamation travailliste sans fondements, mais qui face à une contestation volontairement déficiente est jugée admissible. On simule l'existence d'une dette travailliste seulement pour que le propriétaire de l'entreprise puisse, à travers un accord avec les employés sauver son patrimoine en cas de faillite imminente. ${ }^{6}$

Pontes de Miranda affirme, avec la précision qui lui est particulière, que « dans la simulation, on veut ce qui n'apparait pas et on ne veut pas ce qui apparait» ${ }^{7}$. Vraiment, dans les exemples cités au paragraphe antérieur il apparait que l’employeur/ réclamé ne «veut» pas payer la dite-dette (qui n'existe même pas) à son employé/réclamant: il veut, en fait, récupérer la majeure partie de cette valeur, under the table. De la même façon, le débiteur insolvable qui transfère ses biens à des personnes interposées ne désire pas non plus les vendre ou les donner: il veut les garder, mais les maintenir à l'écart des créditeurs.

Dans ces cas, du fait que les affaires effectivement pratiquées ont une nature «a» (affaire dissimulée, ou occultée), malgré le fait qu'elles simulent une nature «b» (affaire simulée ou apparente), les effets de l'affaire «a» l'emportent, celui effectivement pratiqué.

Nous pouvons dire, au sujet des «pédalages» (acrobaties comptables), qu'ils ont été un emprunt simulé, étant passible, en conséquence, de recevoir le traitement juridique d'un emprunt (une affaire que l'on prétend cacher), et non pas celui d'un «simple retard d'obligation légale» (figure apparente). L'article 167 du Code Civil Brésilien, d'ailleurs, soulève qu'»une affaire juridique simulée est nulle, mais elle substituera ce qu'elle a dissimulé, en cas de validité en substance et en forme».

II y a aussi le cas de figure d'abus de droit, prévu dans l'article 187 du Code Civil Brésilien, qui décide : «commet un acte illicite le titulaire d'un droit qui, dans l'exercice de sa fonction, excède manifestement les limites imposées par son but économique ou social, par la bonne-foi et les bonnes moeurs».

Marco Aurélio Greco rapporte d'intéressants exemples d'abus de droit:

On a créé en France un cas de figure, qui s'appelait une adoption simple, car on exigeait un nombre réduit de conditions pour que les personnes puissent être adoptées. Le but était de faciliter les adoptions. On a établi comme une des conditions le fait que la personne qui adopte soit plus vieille que la personne adoptée, mais on n'a pas spécifié

$6 \quad$ Ce fut avec le but justifié d'éviter ce genre de simulation, d'ailleurs, que la législation brésilienne sur la faillite a été altérée, pour que les crédits travaillistes soient seulement considérés prioritaires jusqu'à la limite de 150 salaires minimums.

7 MIRANDA, F. C. Pontes de. Tratado de Direito Privado. 3 ed. Rio de Janeiro: Borsoi, 1970, v.l, §17, n.8, p. 53. 
(exactement parce qu'on voulait faciliter et agiliser I'adoption) si ce pouvait être une personne de 22 ans avec une autre de 14 et ainsi de suite. Qu'arriva t- il ? Masson cita deux cas levés aux tribunaux dans lesquels l'exercice du droit d'adoption fut considéré abusif, car l'adoption fut considérée comme un instrument pour viabiliser des couples incompatibles; Le premier fut le cas d'un homme marié de 75 ans qui adopta sa concubine de 25 ans, car ainsi lui seraient assurés des droits héréditaires. II ne pourrait pas se marier avec la concubine et a utilisé l'adoption pour obtenir un certain but qui est le propre du mariage; on s'en aperçut et cela généra un procès. Le second cas dont il parle est celui d'un couple d'homosexuels dans lequel le plus vieux adopta le plus jeune pour s'assurer également de droits patrimoniaux.

Quelle est l'importance de ces exemples? Quand on parle d'adoption, on pense immédiatement à un cas de figure de protection d'un enfant, dans le besoin, un orphelin; mais quand on commence à seulement voir le cas de figure légal et que l'on cherche à dire $q u$ 'il existe un "droit d'adopter» à travers lequel il devient possible d'assurer un droit héréditaire pour une personne plus jeune( le cas de figure qui était inscrit dans la loi), en exerçant le droit d'adopter l'agent peut agir contre son profil objectif et on déclarera donc l'abus dans I'exercice du droit. ${ }^{8}$

Ainsi, même si l'Union avait le «droit» de retarder le paiement des ressources aux banques publiques, la forme avec laquelle cela arriva, dans le Gouvernement de Dilma Roussefff, fut totalement contraire au profil objectif du système légal de paiement au moyen des banques publiques et de la rémunération de possibles retard dans le paiement des ressources, la transformant en un contrat d'emprunt interdit par la Loi de Responsabilité Fiscale.

Mais il existe, encore, le cas de figure de la fraude devant la Loi, peut-être le plus pertinent pour ce cas. II se configure quand une affaire est pratiquée dans le but de frauder une norme impérative (CC, article 166, VI). Bien qu'on utilise I'expression «fraude» devant la Loi, le cas n'est pas à confondre avec la fraude proprement dite, qui consiste à occulter ou à faire de fausses déclarations pour les faits pratiqués. Dans la fraude devant la loi, l'agent utilise une forme licite, mais pour atteindre des buts illicites. C'est le cas du père qui, ne pouvant pas vendre un immeuble au fils, le vend à un voisin, qui alors le vend au fils en question ${ }^{9}$. Ce cas de figure, comme il est rapporté, est peut-être celui qui s'ajuste le mieux à la forme par laquelle les acrobaties comptables

\footnotetext{
8 GRECO, Marco Aurélio. Planejamento Fiscal e Interpretação da Lei Tributária. São Paulo: Dialética, 1998, p. 80 .

9 «La fraude devant la loi consiste, donc, à pratiquer un acte de telle manière qu'il puisse éventuellement être appliqué une autre règle juridique en n'appliquant plus la règle juridique fraudée. Celle-ci n'a pas eu d'incidence, parce que celle-là en a eu une; la fraude devant la loi met devant le juge le support des faits, de telle manière que le juge peut commettre une erreur. La fraude devant la loi est une infraction à la loi, l'infracteur
} 
ont été faits, car c'est grâce à eux que l'on a contourné la norme impérative de la Loi de Responsabilité Fiscale qui interdit la contractation d'emprunts auprès des banques publiques contrôlées par la propre entité politique qui sollicite l'emprunt.

Et, comme telle, le pédalage (acrobaties comptables) reste offensif à la LRF, puisque, comme l'explique Pontes de Miranda, «a fraus legis n'est pas opposée à l'agere contra legem; elle est une espèce de celui-ci: jusqu'où arrivera le contenu révélé de la règle juridique, les violations directes et indirectes sont ainsi possibles.» ${ }^{10}$

Quant à l'argument selon lequel le crime de responsabilité serait la violation de la loi financière, et non pas de la loi de responsabilité fiscale, on peut dire, par opposition, que la violation de l'une a des reflets immédiats et directs sur l'autre. En s'autofinançant de forme illégitime et illimitée, la Présidence de la République a transformé la propre loi de finances en une pièce ornementale, car il ne serait pas nécessaire de suivre des recettes, des dépenses et des limites ici prévues dans la mesure où des milliards en obligations appartenant à I'Union ont été jetés sur le dos des banques publiques. Cependant, comme il s'agit d'un grave et dangereux processus de mise en accusation, on peut également soutenir que la pratique du «pédalage» (acrobaties comptables), même étant indéfendable et contraire au droit financier, ne devrait pas permettre la perte du poste par la Présidente. Du point de vue technique, donc, les deux conclusions sont défendables, les arguments politiques assumant de l'importance qui, dans les termes de la Constitution, font que le jugement doive se situer dans la sphère du Sénat Fédéral, et non dans celle du Suprême Tribunal Fédéral, qui serait compétent dans I’hypothèse de crimes communs.

\section{LES DÉCRETS D OUVERTURE DE CRÉDITS SUPPLÉMENTAIRES}

L'ouverture de crédits supplémentaires sans appui dans la loi de finances est exprimée et clairement définie comme un crime de responsabilité par l'art. 10, item6, de la loi 1.079/50, qui définit de tels crimes et apporte un contrôle dans le processus d'impeachment. La question, sur ce point, réside dans le fait que de tels décrets, même ayant été édités dans un manque de respect à de telles conditions, ont été ensuite convalidés par l'altération dans les propres conditions.

Comme il a déjà été expliqué, la Loi de Finances Annuelle (LOA), en 2014 comme en 2015, disait que des crédits supplémentaires pourraient être réalisés, à la condition que l'objectif de l'excédent primaire prévu par la Loi des Directives Financières (LDA), laquelle établit des normes générales et des objectifs de moyen-terme qui doivent être

étant dans l'attente d'une erreur du juge.» MIRANDA, F. C. Pontes de. Tratado de Direito Privado. Atualizado por Vilson Rodrigues Alves. Campinas: Bookseller, T. I, 1999, p. 98.

10 MIRANDA, F. C. Pontes de. Tratado de Direito Privado. 3.ed. Rio de Janeiro: Borsoi, 1970, v. I, §17, n. 8, p. 41. 
suivis dans les deux années respectives (2014 et 2015) ${ }^{11}$. Si l'objectif de l'excédent n'est pas atteint, l'Exécutif ne serait plus autorisé à réaliser de tels crédits. Ce qui s'est passé en 2015, cependant, fut l'édition de tels décrets, sans une autorisation du Législatif (puisque l'objectif n'avait pas été atteint), mais, ensuite, la même année, le Congrès National altéra la Loi des Directives Financières de manière à altérer l'objectif, ce qui aurait légitimé de tels décrets. Enfin, devant une nouvelle prévision d'objectifs, sa réalisation aurait eu lieu, car l'Exécutif serait autorisé à éditer les décrets et à ouvrir des crédits supplémentaires.

Au cas où l'on comprendrait que l'inobservance de l'objectif initial a eu lieu à cause d'une imprévisible crise économique, l'argument de défense de la Présidente apparaitrait comme étant plus raisonnable. De fait, la chose paraitrait assez incohérente si, devant une crise qui mène à la non-réalisation de l'objectif, le gouvernement ne puisse pas utiliser un instrument qui lui servirait exactement dans une telle situation extraordinaire. II est possible d'objecter, toutefois, que l'objectif n'a pas été atteint, principalement, à cause d'un manque de contrôle des dépenses publiques, un manque alimenté par le «pédalage» (acrobaties comptables) et par un excès de dépenses, ce qui peut faire perdre une partie de sa consistance à l'argumentation de défense de Dilma.

II convient de relever, à propos, que l'art. 9 de la Loi de Responsabilité Fiscale (LRF-LC 101/2000), non plus respecté par le Gouvernement Dilma, établit que «s'il est vérifié, à la fin d'un bimestre, que la réalisation de la recette pourra ne pas comporter l'observance des objectifs de résultat primaire ou nominal établis dans I'Annexe des Objectifs Fiscaux, les Pouvoirs et le Ministère Public exécuteront, par un acte propre et de montants nécessaires, dans les trente jours subséquents, une limitation d'intérêt et de transaction financière, en accord avec les critères fixés par la loi des directives financières». En d'autres termes, la Présidente aurait dû vérifier que les objectifs ne seraient pas atteints, en limitant les dépenses publiques avec une contingence de dépenses, ce qui n'arriva pas: elle a préféré éditer les décrets en créant les crédits supplémentaires, de façon à aggraver encore plus le déséquilibre des comptes publics. Dans cet ordre d'idées, la jurisprudence du Tribunal des Comptes, qui avait admis en d'autres occasions que l'altération postérieure de l'objectif fiscal convaliderait des décrets édités avant elle, pourrait être considérée inapplicable parce que, dans ce cas, différemment des antérieurs, le non-respect de l'objectif viendrait déjà d'autres manquements et il devenait prévisible depuis un certain temps.

\footnotetext{
11 Au Brésil, la finance publique est contrôlée par trois strates ou niveaux législatifs, du point de vue temporal. Le Plan Pluriannuel (PPA) définit les objectifs de longue durée à être suivis dans un intervalle de quatre années. Ensuite, la Loi des Directives Financières détaille et rend plus spécifique ces prévisions, pour un espace temporel de deux années. Et, finalement, chaque année, la Loi de Finances Annuelle (LOA) décide sur le budget pour chaque exercice déterminé.
} 
Quant au fait que l'objectif ait été revu par le propre Congrès, nous pouvons dire que celui-ci, en procédant ainsi, a convalidé de fait les décrets. S'il n'était pas d'accord sur la révision de l'objectif, il ne l'aurait pas approuvé. Cependant, il serait encore permis de défendre que, même face à la révision, eux, les décrets, auraient seulement pu être édités si et quand celle-ci serait approuvée, ce qui n’a pas été le cas.

\section{PRINCIPE DE LA COMPÉTENCE, CARACTÈRE PUBLIC DE LA DÉ- CISION ET POSSIBILITÉ DE CRITIQUE DE LA DÉCISION PRISE PAR UN ORGANISME COMPÉTENT}

Nous observons, aussi bien en ce qui concerne le «pédalage» (acrobaties comptables), comme pour ce qui concerne l'ouverture de crédits supplémentaires, que les imputations faites à la Présidente ne se trouvent pas dans une zone de claire non-configuration du crime de responsabilité fiscale, mais elles ne configurent pas non plus de forme indubitable le crime en question. Du point de vue technique, strictement juridique, peut-être nous trouvions-nous devant ce que Hans Kelsen appelle un «cadre ou un tableau» ${ }^{12}$ de significations possibles, le choix de tout un chacun étant scientifiquement correct, devant être guidé par des considérations d'ordre politique. Même si l'existence de cette faculté discrétionnaire ou liberté de l'interprète, défendue par les plus divers courants du positivisme juridique, pour ce qui concerne les décisions juridiques en général, la possibilité voire même la légitimité de sa présence dans le processus d'impeachment semble ne pouvoir être niée.

La raison n'est pas différente, à propos, pour laquelle le jugement du Président de la République pour la pratique de crimes, même s'il doit être l'objet d'une autorisation de la part de la Chambre des Députés, est pratiqué par le Suprême Tribunal Fédéral, dans le cas de crimes communs, mais par le Sénat Fédéral, s'il s'agit de crimes de responsabilité, qui dans ce cas n'a ses séances conduites par le Président du Suprême Tribunal Fédéral qu'exceptionnellement.

Pour cela même, les maintes fois dans lesquelles il fut incité à se manifester sur le procès, le Suprême Tribunal Fédéral s'est prononcé sur le rite à être suivi, sur les organismes devant se manifester, notamment en lieu de jugement de recevabilité, mais il n'a à aucun moment admis pénétrer dans l'essai en soi du mérite, qui doit être apprécié, par respect sur ce que décide la Constitution Fédérale, par le Sénat de la République, comme cela de fait s'est passé.

12 KELSEN, Hans. Teoria Pura do Direito. Tradução de João Baptista Machado. 6. ed., 4. t. São Paulo: Martins Fontes, 2000, p. 369. 
En effet, c'est en appréciant l'audience à huis-clos d'Inobservance du Précept Fondamental (ADPF) $)^{13} 378$, que le Suprême Tribunal Fédéral a compris que quelques-unes des dispositions de la Loi 1.075/50 n'auraient pas été reçues, car elles ne seraient pas compatibles avec la forme avec laquelle la Constitution Fédérale de 1988 contrôle le sujet. Il a ainsi décidé qu'il appartient à la Chambre des Députés, par une majorité qualifiée, de délibérer pour l'instauration du procès. Dès qu'il est autorisé, le procès débute au Sénat, auquel il appartient de décider de sa recevabilité, et, ensuite, de son mérite, la démission pour 180 jours du Président étant prévue par l'article $86, \S 2 .{ }^{\circ}$, de la CF/88, applicable seulement après l'admission du procès par le Sénat.

Par la suite, appréciant le MS 34.131-MC, le Suprême Tribunal Fédéral s'est décidé pour l'absence de nécessité d'une nouvelle manifestation de la défense de la Présidente, face aux éclaircissements faits au sujet de la dénonciation, considérant que la Chambre pourrait seulement autoriser le traitement, la défense étant exercée, de forme ample, devant le Sénat Fédéral, au moment du jugement en question. À travers le MS 34.127, le STF quant à lui s'est prononcé sur la validité de la forme adoptée par le Président de la Chambre des Députés quant au comptage des votes, à la Réunion Plénière de la Chambre, au sujet de l'autorisation pour l'instauration du procès, après avoir adopté un critère pour l'alternance des députés de différentes régions du pays ${ }^{14}$.

Encore une fois pendant la séquence des tentatives, faites par la Présidente et par ceux qui la défendaient, de «judiciariser» le processus d'impeachment, de nombreux habeas corpus ont été sollicités (v.g., HC 134.315) dans lesquels on soutenait que le propre procès représentait un embarras illégal fait à la Présidente, vu que les conduites lui étant imputées ne caractériseraient pas un crime de responsabilité. Cela fut une occasion majeure de la part du Suprême Tribunal Fédéral pour décider la question, entrant dans le vif du sujet et affirmant, si c'était le cas, l'inexistence d'un crime. La cour a compris, cependant, que la question serait de la compétence du Sénat Fédéral, et non de la sienne, étant certain que l'habeas corpus n'aurait aucun sens justement parce qu'il ne s'agit pas d'un crime commun, qui puisse mettre en péril la liberté d'aller et venir de la patiente. Dans le cas de l'impeachment, il y aurait un risque dans l'exercice des droits politiques de la Présidente, non pas pour sa liberté d'aller et de venir, raison pour laquelle I'habeas corpus a été rejeté.

L'idée en est venue, la même défendue il y a longtemps par Themistocles Cavalcanti, selon laquelle l'impeachment ne constitue pas « un institut de droit pénal mais

\footnotetext{
13 À travers la ADPF le contrôle concentré de constitutionnalité des lois éditées antérieurement à la Constitution Fédérale devient possible, comme c'est le cas de la Loi n 1.075/50. On voit, à propos, la Loi n 9.882/99, article $1^{\circ}$, paragraphe unique, incise $\mathrm{I}$.

14 On dit que, avec cela, Eduardo Cunha, alors Président de la Chambre des Députés, prétendait éviter un «effet boule de neige» découlant du fait que, les parlementaires de la région dans laquelle la Présidente aurait plus de votes en sa faveur (v.g., Nord-est du Brésil), la formation d'un score initial lui étant favorable pourrait induire les parlementaires suivants à voter dans le même sens.
} 
bien un acte administratif équivalent à la démission, dont la principale raison est d'écarter des postes importants les fonctionnaires incapables de les exercer dignement» ${ }^{15}$

La décision du Sénat, comme il arrive aux décisions du Suprême Tribunal Fédéral en ce qui concerne des questions essentiellement juridiques, peut, naturellement, être critiquée. C'est ce qui arrive, du reste, aux décisions publiques en général, à partir du moment où elles sont prises par celui, d'après la Constitution, qui est compétent pour cela. Dans le cas de l'impeachment, la compétence pour décider a été attribuée au Sénat Fédéral, qui, l'ayant exercée dans les termes de la Constitution, ne peut être considéré comme étant l'auteur d'un «coup d'état» pour l'unique raison, dans l'esprit de celui qui formule cette imputation, d'avoir adopté une compréhension erronée sur le mérite du jugement.

Quant aux critiques, et aux tentatives de judiciarisation, la dernière découle du fait que le Sénat a réalisé deux votes différents, ayant considéré comme étant possible le fait de condamner la Présidente pour la pratique d'un crime de responsabilité, lui appliquant la peine de perte du mandat, mais non pas celle d'invalidité pour l'exercice de fonctions publiques pendant huit années. On peut critiquer cette décision, car la Constitution ne permettrait pas l'application d'une peine sans l'autre, cela dû à l'utilisation du mot «avec» dans le paragraphe unique de son article 52, selon lequel, dans le cas d'une accusation de crime de responsabilité, "fonctionnera comme président celui du Suprême Tribunal Fédéral, se limitant à la condamnation, qui sera seulement proférée par les deux tiers des votes du Sénat Fédéral, à la perte du mandat, avec invalidité, pendant huit années, pour l'exercice de la fonction publique, sans préjudice dû aux autres sanctions judiciaires appropriées.

II nous semble que le dispositif pense à une punition maximale, la perte du mandat avec une invalidité durant huit années pour l'exercice de fonctions publiques, permettant à l'organisme compétent- le Sénat- de décider pour la condamnation avec l'application d'une sanction inférieure à la maximale, à l'exemple seulement de la perte du mandat, ou de l'invalidité pour une période inférieure à huit ans. Ceci étant, cette question a été soumise à l'appréciation du STF, qui n'a pas encore rendu sa décision au moment de la conclusion de ce travail (MS 34.378).

Quant aux critiques, comme on dit, le processus de mise en accusation peut certainement être leur objet. En commençant par le rôle du Vice- Président, Michel Temer, qui semblait conspirer, auprès du parlement, pour que Dilma Rousseff soit condamnée. Ce n'est certainement pas le rôle d'un Vice-Président, ce qui nous a fait nous remémorer, au Brésil, du personnage de Francis Underwood, de la Série House of Cards, de l'entreprise de streaming de vidéo Netflix. En 1992, le Brésil a assisté, peu après le

15 CAVALCANTI, Themistocles Brandão. A Constituição Federal Comentada. Rio de Janeiro: José Konfino, 1948 , v. II, p. 271. 
rétablissement de la démocratie, à l'impeachment de Fernando Collor de Mello, son premier président élu de forme directe après la fin de la dictature militaire (1964-1986). Dans le cas de Collor, bien que le rite ait été plus rapide et qu'une version contradictoire et une ample défense n'aient pas été assurées de forme si adéquate (comme lui-même l'a fait remarquer, en votant, en tant que Sénateur, en 2016, pour l'impeachment de Dilma), Itamar Franco, son Vice, dont le public s'est rappelé seulement après l'évincement de Collor, n'a pas conspiré contre lui.

Toujours quant à Temer, on ne peut oublier qu'il a, dans la condition de Vice-Président de Dilma, aussi signé des actes d'ouverture de crédits supplémentaires, par lequel, si le motif de l'impeachment était celui-là, le sien devrait également être reconnu. Tout cela montre que, à côté des considérations juridiques et techniques, il existe des aspects politiques qui ont influencé le jugement, quelques-uns légitimes et confessables, comme la délicate situation dans laquelle l'économie du pays a été mise, à cause du déséquilibre dans les comptes publics causé par la gestion de Dilma Rousseff, mais pas pour d'autres.

Mais nous ne pouvons nous empêcher de signaler, par ailleurs, que, comme on l'a dit, le Président Collor de Mello a fait la même expérience, en 1992, ayant été le premier président élu au Brésil, de forme directe, après une longue période de dictature militaire. Collor est tombé à cause d'infractions bien moins graves que celles imputées aujourd'hui à Dilma, et le processus d'impeachment, par rapport à celui-là, fut bien moins obédient à des garanties du vrai processus légal. D’ailleurs dans le jugement rendu par le Suprême Tribunal Fédéral, il a été déclaré innocent, pour ce qui est en rapport avec les infractions communes liées au crime de responsabilité qui ont mené à sa destitution par le Sénat. Encore par rapport à lui, les secteurs de la politique brésilienne qui aujourd'hui crient au « coup d'État» contre Dilma Roussefff ont été pour l'impeachment, à une époque où la démocratie brésilienne faisait encore ses premiers pas. Cela indique peut-être que l'inspiration de telles attributions ne soit simplement que de nature idéologique, car elles n'ont point été utilisées à un moment où elles seraient d'une plus grande pertinence.

À l'époque du précédent impeachment, cependant, la société semblait plus unie pour évincer le président. Malgré les très nombreuses marches réclamant la fin de la corruption juste avant la réalisation de l'impeachment de la Présidente Dilma, de nombreux mouvements en faveur de sa gestion et rejetant sa sortie ont aussi été réalisés.

Avec l'impeachment, en outre, il y eut un changement évident dans le profil politique du gouvernement, qui alla de mesures plus socialistes, vers un gouvernement plus austère. Cette critique, toutefois, qui a trait à l'austérité mise en pratique par l'actuel gouvernement de Michel Temer, n'est peut-être pas seulement fruit d'une idéologie, mais bien d'une nécessité, considérant les dépenses élevées réalisées pendant le 
précédent gouvernement, si nombreuses qu'on a dû utiliser des ressources de banques publiques, afin de permettre le paiement d'obligations publiques.

La gouvernabilité a été également prise en considération pendant l'impeachment. À un certain moment, la situation a semblé incontournable, et la Présidente a commencé à perdre sa légitimité qui lui permettrait d'approuver, au Congrès, des mesures essentielles pour la conduite du pays ${ }^{16}$. Après la fin du processus d'impeachment, la présidente a tenté d'annuler la décision du Sénat, avec l'argument qu'elle n'avait pas perdu son mandat pour cause de pratique de crime de responsabilité, que les Sénateurs n'auraient pas dûment examiné la question. Le ministre du Suprême Tribunal Fédéral rapporteur de l'affaire a été clair en affirmant:

Il est nécessaire de relever que, de par la qualité essentiellement politique des crimes de responsabilité, la projection attentatoire à la Constitution Fédérale ne se détachera pas, le plus souvent, de l'acte unitairement imputé à l'accusé, mais de la désinvolture négative que celui-ci acquiert dans le contexte de gouvernance globale de l'Administration Publique ${ }^{17}$.

Toutes les institutions ont fonctionné de manière formelle, il n'a pas été nécessaire d'utiliser des canons dans les rues pour contenir des révolutions, mais il faut reconnaitre que la démocratie brésilienne est dans une période sensible, avec une augmentation de l'agressivité chez les citoyens. Sur ce point, il est important de rappeler les leçons de Simone Goyard-Fabre selon lesquelles les vertus de la démocratie sont en même temps ses faiblesses ${ }^{18}$. Des vagues de périodes tranquilles sont suivies par des moments de tensions, dans des successions aléatoires. Il importe que l'opportunité du vote soit maintenue, ainsi que les libertés de pensée et d'expression, avec en outre l'accès à l'information.

\section{CONCLUSIONS}

Le processus d'impeachment de Dilma Rousseff, au Brésil, découle de l'adoption de conduites questionnables à la lumière du Droit Financier, qui ont mené à un déséquilibre des comptes publics, avec des conséquences assez nuisibles pour l'économie nationale, lesquelles sont ressenties, dans le secteur public et l'initiative privée, jusqu'à nos jours, pouvant continuer durant de nombreuses années.

\footnotetext{
16 Et c'est naturel au processus : "Hamilton deduced that impeachment involves not only the various constitutional actors (the House, the Senate, the chief justice of the Supreme Court, and the president) but also the two great evils the Founders feared most: faction and the unregulated passions of the people." Cf. SILVA, Carol; JENKINS-SMITH, Hank; WATERMAN, Richard. Why Did Clinton Survive the Impeachment Crisis? A Test of Three Explanations. Presidential Studies Quarterly, Washington, v. 37, n. 3, p. 468-485, Sep. 2007, p. 470.

17 MS 34.371

18 FABRE, Simone Goyard. O que é Democracia? São Paulo: Martins Fontes, 2003, p. 342.
} 
On ne peut nier, malgré tout, l'aspect polémique et limitrophe de l'encadrement de ces ilégalités comme crime de responsabilité fiscale, raison pour laquelle on ne peut qualifier d'absurdes ou d'inopportunes les points de vue de ceux qui reconnaissent la présence de conditions techniques pour l'impeachment, comme de ceux qui les rejettent.

Sur le plan politique, l'existence de motifs qui justifient les deux décisions - celle de l'écarter et celle de maintenir la présidente - est encore plus éloquente. Si, d'un côté, ses actions ont conduit l'économie vers une situation difficile et, du point de vue politique, la gouvernabilité et la stabilité des institutions étaient précaires, notamment en vertu de la participation de membres hiérarchiquement importants de son gouvernement dans des scandales de corruption, d'un autre côté, les Députés et Sénateurs qui ont voté en faveur de son impeachment sont, la plupart d'entre eux, dans des situations encore plus complexes, d'un point de vue éthique, pour ce qui concerne la participation à des scandales de corruption, comme c'est le cas du président de la Chambre des Députés, Eduardo Cunha, qui aussitôt après a perdu son poste pour cause de mensonges liées à la titularité de comptes à l'étranger alimentés par des ressources provenantes de pots de vin, et de tant d'autres parlementaires, de nombreux autres partis, pris par les mêmes scandales de corruption qui ont consommé la crédibilité du Parti de Dilma Rousseff. II s'ajoute à cela le fait que Michel Temer, son Vice-Président, ait assumé la posture d'adhérer à la thèse de l'impeachment, conspirant politiquement peut-être pour faciliter sa finalisation, en dehors du fait d'avoir signé, autant que Dilma, des décrets pour la création de crédits supplémentaires ${ }^{19}$.

De quelque manière que ce soit, le fait que la décision ait été prise par l'organisme compétent pour cela, sous les lumières du texte constitutionnel, avec le contrôle, en ce qui concerne les aspects formels, du Suprême Tribunal Fédéral, et à partir des constatations du Tribunal des Comptes et du Ministère Public des Comptes, nous montre qu'il n'est pas approprié d'affirmer qu'il y a eu un «coup d'État», vu que tout s'est passé dans les termes prévus par le texte constitutionnel.

Les critiques pouvant être dirigées au mode opératoire, même si elles sont légitimes, n'autorisent pas sa comparaison avec une rupture des institutions démocratiques ni avec un manque de respect pur et simple envers la Constitution.

\footnotetext{
19 Peut-être à cause de cela, Malcolm Parsons considère que l'impeachment, à la lumière de l'histoire américaine, est "often highly political and ineffectual". Pour lui, il est préférable d'utiliser des instituts tels que la réélection (avec la possibilité que le titulaire ne soit pas réélu), ou le recall. Cf. PARSONS, Malcolm. Checking abuse of power: does impeachment work? National Civil Review, [s.l.], p. 219-224, jui./août 1986, p. 220 e ss.
} 


\section{RÉFÉRENCES}

ARATO, Andrew. Impeachment or revision of the Constitution? Constellations, Massachusetts, v. 6, n.2, 145-156, 1999.

BONAVIDES, Paulo. Ciência Política. 10 ed. São Paulo: Malheiros.

CAVALCANTI, Themistocles Brandão. A Constituição Federal Comentada. Rio de Janeiro: José Konfino, 1948, v. II.

FABRE, Simone Goyard. 0 que é Democracia? São Paulo: Martins Fontes, 2003.

GRECO, Marco Aurelio. Planejamento Fiscal e Interpretação da Lei Tributária. São Paulo: Dialética, 1998.

KELSEN, Hans. Teoria Pura do Direito. tradução de João Baptista Machado. 6. ed., 4. t. São Paulo: Martins Fontes, 2000.

MIRANDA, Pontes de. Tratado de Direito Privado. 3.ed., Rio de Janeiro: Borsoi, 1970, v. I, § 17, n. 8. MIRANDA, Pontes de. Tratado de Direito Privado. Atualizado por Vilson Rodrigues Alves. Campinas: Bookseller, Tomo I, 1999.

PARSONS, Malcolm. Checking abuse of power: does impeachment work? National Civil Review, [s.l.], p. 219-224, jui./août 1986.

RIBEIRO, Ricardo Lodi. Parecer sobre a acusação de crime de responsabilidade contra Dilma Rousseff. Disponible dans: <http://s.conjur.com.br/dl/parecer-ricardo-lodi-impeachment.pdf>. L'accès à: 6 dez 2019.

SILVA, Carol; JENKINS-SMITH, Hank; WATERMAN, Richard. Why Did Clinton Survive the Impeachment Crisis? A Test of Three Explanations. Presidential Studies Quarterly, Washington, v. 37, n. 3, p. 468-485, Sep. 2007. 